Revista de

Contabilidade e

Organizações

www.rco.usp.br
DOI: http://dx.doi.org/10.11606/issn.1982-6486.rco.2019.158510
Journal of

Accounting and

Organizations

\title{
Corrupção, lavagem de dinheiro e conluio no Brasil: evidências empíricas dos vínculos entre fraudadores e cofraudadores no caso Lava Jato
}

Corruption, Money laundering schemes and collusion in Brazil: empirical evidence of the links between fraudsters and co-offenders at "Lava Jato" Operation

Lucas Martins Dias Maragno ${ }^{a}$, Paulo de Souza Knuppa, José Alonso Borba ${ }^{a}$

${ }^{a}$ Universidade Federal de Santa Catarina

Palavras-chave

Corrupção.

Lavagem de dinheiro.

Cofraudadores.

Lava Jato.
Keywords

Corruption.

Money laundering schemes.

Co-offenders.

Lava Jato.
Informações do artigo

Recebido: 30 de maio de 2019

Aceito: 14 de novembro de 2019

Publicado: 28 de novembro de 2019

\section{Resumo}

Este estudo exploratório objetiva compreender os vínculos que conectam cofraudadores em esquemas de corrupção e lavagem de dinheiro presentes na Operação "Lava Jato". A base de dados foi construída por meio da leitura das denúncias realizadas e documentadas pelo Ministério Público Federal (MPF). Foram identificadas todas as pessoas acusadas pelo MPF e suas características (gênero, idade e experiência anterior ao crime), assim como a natureza dos vínculos entre fraudadores e cofraudadores, o tipo de crime e o papel do acusado no crime. Sinteticamente, os resultados demonstraram que os fraudadores líderes já haviam sido condenados anteriormente e/ou possuíam experiência nas fraudes cometidas. Os resultados demonstraram ainda que os vínculos afetivos estão positivamente relacionados à apropriação indébita, ocultação de provas e "testa de ferro". Se por um lado, Fee e Murphy (2015) destacaram a natureza dos vínculos entre membros de grupos fraudulentos, nossos achados evoluem e fornecem algumas evidências empíricas dos vínculos funcionais que servem à organização e invariavelmente para os vínculos afetivos.

\begin{abstract}
This exploratory study aims to understand the bonds that connect co-offenders in corruption and money laundering schemes at "Lava Jato" Operation. The database was built by reading the denunciations made by the Federal Public Ministry (MPF). All the accused persons, and their characteristics (gender, age and pre-crime experience) have been identified, as well as the nature of the type of connection between fraudsters and co-offenders, type of crime and role in crime. The results have shown that the leaders are those who have previously been convicted and/or have experience with the frauds. Regarding the nature of the bonds, the results highlighted that the affective bonds are positively related to misappropriation, concealment of evidence and being a straw man. In one hand, Fee and Murphy (2015) have highlighted the nature of the bonds between members of fraudulent groups, our findings evolve and provide some empirical evidence for the organization-serving functional bonds and invariably for affective bonds.
\end{abstract}

\section{Implicações práticas}

Os resultados demonstraram que a corrupção foi realizada não somente por pessoas que possuíam relações de afetividade/amizade, mas também relações de trabalho. Tal fato pode dificultar a identificação de ligações suspeitas entre criminosos pelos órgãos de controle. Neste sentido, seria imprescindível buscar cada vez mais o aperfeiçoamento dos órgãos de inteligência e sistemas de controle considerando as características dos fraudadores e cofraudadores e suas relações, ambas abordadas neste estudo. 


\section{INTRODUÇÃO}

Corrupção e lavagem de dinheiro estão intrinsecamente conectadas, pois muitas vezes ocorrem simultaneamente (Chaikin, 2008; Cox, 2014; OCDE, 2018). Cox (2014) explica que a lavagem de dinheiro começa com a atividade criminosa, onde se originam os recursos ilícitos, e menciona que tanto a evasão fiscal quanto a corrupção resultam na produção de recursos que o fraudador tentará disfarçar (ou lavar). A corrupção, como crime, é o esquema mais comum em todas as regiões do mundo, e tem fortes impactos no desenvolvimento econômico e social. Estima-se que ela absorve de 1,5 a 2 trilhões de dólares anualmente com impacto negativo para o crescimento econômico, geração de perdas com receitas tributárias e promoção da pobreza (Reuters, 2016; Dimant \& Tosato, 2018; ACFE, 2018).

Assim como a corrupção, a lavagem de dinheiro é considerada significativa e global. O escritório das Nações Unidas sobre Drogas e Crime estima que entre 2\% e 5\% do PIB mundial é "lavado" a cada ano (UNODC, 2019). Isso significa montantes anuais entre 800 bilhões e 2 trilhões de dólares. Exemplos como os casos do Danske Bank, Swedbank, BNP Paribas e HSBC demonstram que a lavagem de dinheiro está presente em diversas partes do mundo, principalmente, no sistema financeiro (Mclannahan, 2017; Milne, 2019; Schwartzkopff \& Magnusson, 2019).

Em grandes esquemas de corrupção e lavagem de dinheiro pode existir um elemento central, que é o conluio (Free, 2015). De acordo com Free e Murphy (2015) é improvável que qualquer um tenha recursos, acesso e capacidade para construir uma fraude sofisticada sem a ajuda de terceiros. Ainda, o conluio potencializa a fraude, tornando-a mais severa, em termos de custos, e ameaça mecanismos antifraude (Trompeter, Carpenter, Jones \& Riley Jr, 2014; Bishop, Hermanson \& Riley Jr, 2017). O relatório da ACFE de 2018 mostra que o conluio entre dois fraudadores tende a ser mais custoso, em média o dobro, do que as fraudes de somente um fraudador e, para 3 fraudadores, a perda aumenta em 4,5 vezes.

Apesar disso, diversos autores apontam uma falta (ou negligência) de pesquisas sobre fraudes que se concentram no conluio (Van Mastrigt \& Farrington, 2011; Trompeter, Carpenter, Jones and Riley Jr, 2014; Free, 2015; Bishop, Hermanson \& Riley Jr, 2017; Button, Shepherd \& Blackbourn, 2018). Isso ocorre porque grande parte da literatura busca compreender as motivações individuais sobre fraudes e não o conluio (ou relações) entre fraudadores (Pinto, Leana \& Pil, 2008; Free \& Murphy, 2015; Maragno \& Borba, 2017). Recentemente, a literatura sobre corrupção sugeriu uma mudança nas pesquisas, da corrupção no nível individual para uma corrupção coletiva (Kominis \& Dudau, 2018). Diante disso, o fenômeno do "co-offending"1 (conluio) e a função dos "cooffenders" (cofraudadores) ainda são pouco compreendidos, principalmente, em esquemas de corrupção e lavagem de dinheiro. Bishop, Hermanson e Riley Jr (2017) apontam que uma exceção à falta de pesquisas sobre conluio é o trabalho de Fee e Murphy (2015).

Ainda, a literatura que trata do conluio é limitada à delinquência juvenil e à crimes específicos (Weerman 2003; Van Mastrigt \& Farrington, 2011; McGloin \& Nguyen, 2012; Lantz \& Hutchison, 2015; Tillyer \& Tillyer, 2015). Dessa maneira, para preencher essa lacuna e investigar aspectos relacionados ao conluio em fraudes, propõe-se analisar as características dos líderes nas fraudes, e se a natureza do vínculo entre fraudadores e cofraudadores está relacionada a tipos específicos de fraudes. O objetivo deste trabalho busca fornecer evidências sobre os vínculos que conectam os fraudadores e os tipos de crimes envolvidos nessas relações, principalmente, em esquemas de corrupção e lavagem de dinheiro. Também, visa contribuir para a compreensão das características dos líderes no conluio.

Assim, as perguntas foram respondidas com base na maior investigação de corrupção e lavagem de dinheiro brasileira, originada pela Operação "Lava Jato". A operação demonstrou que 438 pessoas foram acusadas criminalmente, incluindo políticos e membros da diretoria de empresas como Petrobras, Odebrecht e, outras empresas do setor de construção civil. Nesse sentido, este trabalho contribui para um crescente de pesquisas que teorizam a corrupção como um problema de ação coletiva, principalmente, em sociedades em que a corrupção é o comportamento esperado (Kominis \& Dudau, 2018; Jancsics, 2019). Segundo essa teoria (problema de ação coletiva), a corrupção persiste porque o agente público percebe que todos os outros agentes provavelmente são corruptos, de forma semelhante à teoria da escolha pública - a corrupção é explicada pelo cálculo racional dos meios e fins (Jancsics, 2019).

\footnotetext{
1 "Co-offending" se refere à perpetração de uma fraude por mais de uma pessoa e inclui cooperação criminosa em diferentes momentos e lugares, um processo no qual os indivíduos voluntariamente agrupam seus recursos na busca de objetivos compartilhados, mas ilegais (Free \& Murphy, 2015).

${ }^{2}$ Segundo site do MPF em 21 de setembro de 2019. Fonte: <http://www.mpf.mp.br/grandes-casos/caso-lava-jato/atuacao-na-1a-instancia/ parana/resultado>
} 


\section{CORRUPÇÃO, LAVAGEM DE DINHEIRO E CONLUIO}

Uma das definições aceitas descreve a corrupção como o uso indevido de cargos públicos para ganhos privados (Alvarez, 2015; Kominis \& Dudau, 2018; Gonçalves \& Andrade, 2019). A corrupção pode ser dividida em corrupção de alto nível e corrupção de baixo nível. A primeira é entendida como qualquer atividade corrupta envolvendo altos funcionários administrativos ou políticos (Alvarez, 2015). Isso pode levar à corrupção coletiva, pois estas pessoas têm os recursos e o poder de mudar as "regras do jogo" para obterem ganhos pessoais (Kominis \& Dudau, 2018). Já a segunda envolve a interação entre funcionários públicos de baixa hierarquia e cidadãos comuns, impulsionada por incentivos econômicos (Alvarez, 2015).

Essa divisão advém da tipologia multidimensional, organizada em torno de duas variáveis que consideram os tipos de recursos estatais, bem como os atores em diferentes níveis de governo com controle sobre esses recursos (Jancsics, 2019). Ashforth \& Anand (2003) apontam que a motivação e a oportunidade de se envolver em corrupção depende de fatores ambientais (forte competição, aplicação legal e regulatória), organizacionais (baixo desempenho, complexidade estrutural) e, em menor grau, de fatores pessoais.

Já o processo de lavagem de dinheiro é descrito em três estágios: ocultação, dissimulação e integração (Levi \& Reuter, 2006; Chaikin, 2008; Cox, 2014). Ocultação é a introdução dos recursos ilícitos no sistema financeiro (Levi \& Reuter, 2006). Dissimulação é um conjunto de atividades destinadas a distanciar os recursos ilícitos de seu ponto de origem (Levi \& Reuter, 2006; Chaikin, 2008). E integração é a conversão dos recursos ilegais em ganhos comerciais aparentemente legítimos, por meio de operações financeiras ou comerciais normais (Levi \& Reuter, 2006). Maragno e Borba (2019) demonstram que essas três fases estão presentes no caso Petrobras, a partir de uma análise da primeira fase da Operação "Lava Jato". De forma mais ampla, Gonçalves e Andrade (2019) descrevem o caso "Lava Jato" por meio de uma abordagem sociológica.

A fase de maior vulnerabilidade é a de ocultação (Levi \& Reuter, 2006; Cox 2014). Pois, as instituições financeiras são obrigadas a verificar a identidade do cliente e realizar uma medida de due diligence, aumentando a probabilidade de deteç̧ão do crime (Chaikin, 2008; Cox, 2014). Contudo, prestadores de serviços especializados como, por exemplo, advogados e contadores, possuem conhecimentos e habilidades para criar e organizar esquemas de lavagem de dinheiro e, podem incluir a dissimulação ou a integração de transações financeiras, usando empresas em paraísos offshore, juntamente com a ocultação ou a destruição de registros, evitando a detecção (Chaikin, 2008).

Corrupção e lavagem de dinheiro ocorrem frequentemente em conjunto, com a presença de uma reforçando a outra (Chaikin, 2008). Ainda, a corrupção pode estar presente em todas as etapas da lavagem de dinheiro, mas tem sua maior oportunidade na fase de ocultação, porque indivíduos com poder político que desejam ocultar recursos ilícitos podem subornar os agentes das instituições financeiras para impedir a descoberta de suas contas bancárias (Chaikin, 2008).

As pesquisas sobre conluio têm foco em delinquência juvenil (Weerman 2003; Van Mastrigt \& Farrington, 2011; McGloin \& Nguyen, 2012; Lantz \& Hutchison, 2015; Tillyer \& Tillyer, 2015). Weerman (2003) afirma que o modelo teórico mais abrangente de delinquência conjunta proposto é a "Social Exchange Theory of Co-offending" que é fundamentada nas áreas de psicologia social e sociologia. Essa teoria conceitua o delito em conjunto como uma troca interpessoal de bens materiais e imateriais, na qual cada ofensor tem algo a ganhar com a cooperação do outro. Para Van Mastrigt e Farrington (2011) o recrutamento de coinfratores pode ser um mecanismo importante por meio do qual as definições, habilidades e racionalizações criminais são transmitidas de criminosos mais experientes para criminosos menos experientes.

Por um lado, como benefícios ao conluio, Weerman (2003) sugere que os infratores antecipam que cometer crimes com cúmplices será mais fácil, mais lucrativo e menos arriscado em relação a cometer crimes individualmente. Os infratores percebem os coinfratores como um ativo (Tillyer \& Tillyer, 2015). Por outro lado, os grupos criminosos são menos estáveis, pois seus membros podem ser presos ou desistir ao longo do tempo e, existe o risco de pelo menos um membro do grupo ser preso e subsequentemente expor os demais (Weerman, 2003). Também, a manutenção a longo prazo de grupos criminosos é mais difícil (Lantz \& Hutchison, 2015) e pode haver traição entre membros (McGloin \& Nguyen, 2012).

A literatura em sociologia, psicologia social e criminologia aponta que idade e gênero (Bonny, Goode \& Lacey, 2015), tipo de crime (Button, Shepherd \& Blackbourn, 2018) e experiência sobre crimes (Weerman, 2003) explicam os motivos de alguns indivíduos serem "líderes" ou "instigadores". Esses indivíduos são vistos como aqueles que convencem outros a participarem da delinquência coletiva (Mcgloin \& Nguyen, 2012). Assim, eles emergem por causa de habilidades que são relevantes para a tarefa ou problema em questão, e a experiência criminal pode determinar o status entre os infratores (Weerman, 2003; Mcgloin \& Nguyen, 2012). 
Em relação ao gênero dos fraudadores em fraudes ocupacionais, Bonny, Goode e Lacey (2015) demonstraram que o custo médio estimado da fraude por homens é mais de quatro vezes maior do que aqueles cometidos por mulheres. Ainda, os autores afirmam que em fraudes de colarinho branco, tem-se observado taxas de fraudes mais baixas para as mulheres. Ainda, do ponto de vista teórico, argumenta-se que as mulheres são menos orientadas de forma individual (egoístas) do que os homens (Dollar, Fisman \& Gatti, 2001). No estudo de Dolar, Fisman e Gatti (2001), os resultados demonstraram que as mulheres tendem a adotar posições mais fortes em relação ao comportamento ético.

Os resultados de Bishop, Hermanson \& Riley Jr (2017) demonstraram algumas diferenças entre fraudes em conluio e fraudes individuais em relação às características dos líderes. Os fraudadores líderes são mais jovens e mais propensos a serem do sexo masculino (Kleemans \& De Poot, 2008; Bishop, Hermanson \& Riley Jr, 2017) e, menos propensos a terem diplomas universitários (Bishop, Hermanson \& Riley Jr, 2017).

Bishop, Hermanson e Riley Jr (2017) assinalam o trabalho de Free e Murphy (2015) contribuiu para o desenvolvimento da literatura sobre conluio e, ainda, apontam que a razão para a escassez de pesquisas é a indisponibilidade de dados.

Para contornar tal problema, Free e Murphy (2015) entrevistaram 37 fraudadores que cometeram fraudes em conluio. Os autores concluíram que os motivos pelos quais os fraudadores cometem fraudes variam de acordo com três naturezas qualitativas dos laços entre os membros do grupo: (i) vínculos funcionais que servem ao indivíduo - os cofraudadores encontram em seu próprio interesse individualista cooperar com os outros na busca de benefícios individuais; (ii) vínculos funcionais que servem à organização - baseiam-se em uma afiliação maior no local de trabalho; e (iii) vínculos funcionais afetivos - referem-se a laços baseados em conexões emotivas entre cofraudadores.

Portanto, dado o custo da fraude em conluio e essa limitação de pesquisas sobre o tema, estudar as relações entre fraudadores no conluio pode ser útil para a compreensão do comportamento corrupto dos indivíduos (Pinto, Leana \& Pil, 2008; Bishop, Hermanson \& Riley Jr, 2017).

\section{PROCEDIMENTOS METODOLÓGICOS}

\subsection{Coleta de dados}

Para construção da base de dados foram lidos dez processos apresentados pelo Ministério Público Federal, que compreendem a primeira fase da investigação conhecida como Operação "Lava Jato". A primeira fase é conhecida por ter seu foco na atuação de quatro doleiros - aqueles considerados líderes das organizações criminosas segundo os processos da "Lava Jato". Dessa maneira, segundo Weerman (2003) e Mcgloin e Nguyen (2012), seriam eles os responsáveis por cooptarem outros fraudadores. A Tabela 1 descreve os processos que foram utilizados.

Tabela 1. Denúncias da Operação "Lava Jato"

\begin{tabular}{|c|c|c|}
\hline $\mathbf{N}$ & Descrição dos crimes & Valor total aproximado \\
\hline 1 & Crime de embaraço à investigação de organização criminosa. & - \\
\hline 2 & $\begin{array}{l}\text { Prática de crimes financeiros, mercado paralelo de câmbio e formação de instituição } \\
\text { financeira irregular. }\end{array}$ & R \$ 5 milhões \\
\hline 3 & $\begin{array}{l}\text { Prática de crimes financeiros, de lavagem de dinheiro e de formação de organização } \\
\text { criminosa. Evasão fraudulenta. }\end{array}$ & US\$ 500 milhões \\
\hline 4 & Prática de crimes de tráfico internacional de drogas e lavagem de dinheiro. & US\$ 124 mil \\
\hline 5 & Crimes contra o sistema financeiro e mercado paralelo de câmbio. & R\$ 3 milhões \\
\hline 6 & Crimes de associação criminosa e lavagem de dinheiro. & $\mathrm{R} \$ 25$ milhões \\
\hline 7 & Evasão de divisas e lavagem de dinheiro. & $\mathrm{R} \$ 11$ milhões \\
\hline 8 & Crimes de organização criminosa e contra o Sistema Financeiro Nacional. & $\mathrm{R} \$ 2,5$ milhões \\
\hline 13 & $\begin{array}{l}\text { Crime de lavagem de dinheiro, associação criminosa, apropriação indébita e } \\
\text { estelionato. }\end{array}$ & $\mathrm{R} \$ 10$ milhões \\
\hline 14 & Crimes financeiros e de lavagem de dinheiro. & $\mathrm{R} \$ 215$ milhões \\
\hline
\end{tabular}

Fonte: http://www.mpf.mp.br/para-o-cidadao/caso-lava-jato/atuacao-na-1a-instancia/parana/denuncias-do-mpf.

Nota: Quatro denúncias dessa fase não foram consideradas (processos de 9 a 12) pois tratavam de processos referentes ao caso Banestado, arquivados devido a um acordo de delação premiada em 2000. 
A partir da leitura dos processos, os pesquisadores, primeiro, identificaram as pessoas em foco - aquelas acusadas formalmente pelo Ministério Público. Na sequência, as características dos mesmos foram identificadas, sendo elas: gênero, idade e experiência anterior ao crime - condenado anteriormente ou experiência pelo mesmo crime imputado na investigação, o tipo de crime e o papel no crime. Também, as relações entre os acusados e as pessoas de ligação (i.e cofraudadores) foram tabuladas, assim como as características dos cofraudadores (gênero, idade, experiência anterior ao crime, tipo de crime e o papel no crime do cofraudador). Para ilustrar a relação entre os fraudadores, segue o seguinte exemplo:

\section{“Xjunto com o seu primo Y negociavam venda de dólar com o cliente Z”}

Dessa maneira foram identificadas as relações entre X e Y; X e Z; e Y e Z, o Apêndice A ilustra os vínculos encontrados, assim como exemplos de experiência e papel no crime.

\subsection{Identificação dos dados}

\section{Natureza do Vínculo}

A Natureza do Vínculo registra os laços interpessoais que existem entre os indivíduos denunciados pelo Ministério Público Federal. As naturezas dos vínculos entre membros do grupo criminoso foram classificadas com base nos conceitos de Free e Murphy (2015). Dessa maneira, as relações são descritas na Tabela 2.

Tabela 2. Natureza do Vínculo

\begin{tabular}{clc}
\hline Natureza do Vínculo & Relação & Descrição \\
\hline \multirow{2}{*}{ Vínculos afetivos } & Familiar & Casais, casais divorciados, filhos, irmãos, cunhados, mães, pais, etc. \\
\cline { 2 - 3 } & Amizade & Amigos e compadres. \\
\hline Vínculos funcionais & Profissional & Clientes e prestadores de serviços, funcionários e sócios. \\
\hline
\end{tabular}

Fonte: Elaborada pelos autores a partir de Free e Murphy (2015).

\section{Papel no Crime}

O Papel no Crime, conforme Tabela 3, se refere à função e a posição na hierarquia exercida e ocupada pelo acusado, segundo descrito nos processos apresentados pelo MPF.

Tabela 3. Papel no crime identificado nos processos

\begin{tabular}{|c|c|}
\hline Papel no crime & Descrição \\
\hline Líder & Aquele que lidera, faz o pedido, manda. \\
\hline Operacional & $\begin{array}{l}\text { Aquele que executa atividades operacionais, como saques, depósitos, transporte de dinheiro } \\
\text { e vendas. }\end{array}$ \\
\hline Gerencial & Aquele que executa atividades gerenciais, como administrar empresas/operações. \\
\hline Laranja & Aquele que empresta/dá/vende o nome para ser usado na formação de sociedade de empresas \\
\hline Laranja gerencial & Combinação de gerente com laranja. \\
\hline Laranja operacional & Combinação de operacional com laranja. \\
\hline Doleiro & Aquele operador do mercado paralelo ou negro de câmbio. \\
\hline Traficante & Aquele que movimenta e vende drogas. \\
\hline Político & Aquele que ocupa um cargo público político. \\
\hline Cliente & Aquele que contrata os serviços de um doleiro. \\
\hline Não descreve & $\begin{array}{l}\text { Foi usado esse termo quando os denunciantes não informavam se o sujeito executava alguma } \\
\text { atividade ilegal. }\end{array}$ \\
\hline
\end{tabular}

Fonte: Elaborada pelos autores conforme descrito nos processos do MPF.

Nota: Ressalta-se que um indivíduo com papel gerencial poderia realizar atividades operacionais, contudo foi classificado apenas com a maior função hierárquica. 


\section{Tipo de Crime}

O Tipo de Crime, conforme Tabela 4, se refere ao ato praticado pelos indivíduos citados nas denúncias. Para isso, foi considerada a descrição presente nos processos.

Tabela 4. Tipos de crimes identificados nos processos

\begin{tabular}{|c|c|c|}
\hline $\begin{array}{l}\text { Tipo de Crime descrito no } \\
\text { processo }\end{array}$ & Descrição & Tratamento pela Lei \\
\hline \multirow[b]{3}{*}{ Mercado Negro de Dinheiro } & $\begin{array}{l}\text { Operações realizadas com o intuito de esconder a origem } \\
\text { ilícita do dinheiro. }\end{array}$ & $\begin{array}{c}\text { Art. } 1^{\circ} \\
\text { Lei }{ }^{\circ} 9.613 / 1998\end{array}$ \\
\hline & $\begin{array}{l}\text { Enviar para fora do país dinheiro de forma irregular, seja } \\
\text { por meio físico ou por operações financeiras justificadas } \\
\text { por contratos falsos. }\end{array}$ & $\begin{array}{c}\text { Art. } 22^{\circ} \\
\text { Lei } n^{\circ} 7.492 / 1986\end{array}$ \\
\hline & Agir como instituição financeira no Brasil sem permissão. & $\begin{array}{c}\text { Art. } 16^{\circ} \\
\text { Lei } n^{\circ} 7.492 / 1986\end{array}$ \\
\hline Corrupção & $\begin{array}{l}\text { Atividades ilegais que envolviam a colaboração de } \\
\text { funcionários públicos. }\end{array}$ & 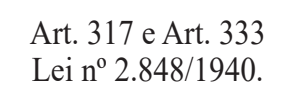 \\
\hline Ocultação de Provas & Suprimiram papéis, documentos e valores de empresas. & $\begin{array}{c}\text { Art. } 2^{\circ} \\
\text { Lei } n^{0} 12.850 / 2013 \text {. }\end{array}$ \\
\hline \multirow[t]{2}{*}{ Apropriação Indébita } & $\begin{array}{l}\text { No caso desse estudo, tomada de posse de uma empresa } \\
\text { sem o consentimento dos sócios. }\end{array}$ & $\begin{array}{c}\text { Art. } 168 \\
\text { Lei } \mathrm{n}^{\mathrm{o}} 2.848 / 1940\end{array}$ \\
\hline & $\begin{array}{l}\text { Uso do nome de uma pessoa para realizar atividades que } \\
\text { beneficiassem o grupo criminoso, tal como abrir uma } \\
\text { empresa de fachada. }\end{array}$ & $\begin{array}{c}\text { Art. } 2^{\circ} \\
\text { Lei }{ }^{\circ} 12.850 / 2013 \text {. }\end{array}$ \\
\hline Tráfico & Movimentação e venda de drogas ilícitas. & $\begin{array}{c}\text { Art. } 33 \\
\text { Lei }{ }^{\circ} 11.343 / 2006\end{array}$ \\
\hline Não descreve & $\begin{array}{l}\text { Termo utilizado quando o processo não informava se a } \\
\text { relação visava uma atividade ilegal. }\end{array}$ & - \\
\hline
\end{tabular}

Fonte: Elaborada pelos autores conforme descrito nos processos do MPF.

\subsection{Modelos lineares generalizados}

Os modelos 1 e 2 foram estimados com as variáveis dependentes Líder e Gerencial. Nesses modelos, o objetivo foi capturar se as características pessoais (isto é, Idade, Gênero e Experiência) explicam as posições na hierarquia dos crimes. As variáveis para os Modelos a seguir estão definidas na Tabela 5 e demonstradas no Apêndice A.

Modelos 1 e 2:

$$
\left(\text { Lider }_{j} ; \text { Gerencial }_{j}\right)=\beta_{0}+\beta_{1} \text { Idade }_{j}+\beta_{2} \text { Gênero }_{j}+\beta_{3} \text { Experiência }_{j}+\varepsilon_{j}
$$

Modelos 3 e 4: $\left(\right.$ VincSig $_{j} ;$ VincAfe $\left._{j}\right)=\beta_{0}+\left(\beta_{1}\right.$ Apro $_{j}+\beta_{2}$ Corr $_{j}+\beta_{3}$ Eva $_{j}+\beta_{4}$ Lava $_{j}+\beta_{5}$ Merc $_{j}+\beta_{6}$ Ocult $_{j}+\beta_{7}$ Test $\left._{j}\right)$ TiposdeCrime
$+\beta_{8}$ PreLider $_{j}+\beta_{9}$ PreGer $_{j}+\varepsilon_{j}$

Já o modelo 3 foi estimado com a variável dependente VincSig com o propósito de capturar o efeito de vínculos significativos entre os fraudadores e cofraudadores (ie. profissional ou afetiva) ou não significativas (ie. não descrito). O modelo 4 foi estimado com a variável dependente VíncAfe, vínculo afetivo (família ou amizade) ou vínculo não afetivo (profissional ou não descrito). Esse procedimento buscou capturar os efeitos dos vínculos afetivos em relação a crimes específicos. Também, buscou-se capturar o efeito a partir da presença de líderes e gerentes, a fim de observar se esses preferem o conluio entre pessoas que tenham vínculos afetivos ou não. 
Tabela 5. Mensuração e descrição das variáveis utilizadas nos modelos

\begin{tabular}{|c|c|}
\hline Variável & Mensuração e Descrição \\
\hline \multicolumn{2}{|l|}{ Dependentes } \\
\hline Líder & 0 = não líder, 1 = líder \\
\hline Gerencial & $0=$ cargo não gerencial, $1=$ cargo gerencial \\
\hline VíncSig & $\begin{array}{l}0 \text { = vínculo não descrito, } 1 \text { = vínculo significativo (profissional, familiar ou } \\
\text { amizade) }\end{array}$ \\
\hline VíncAfe & $\begin{array}{l}0=\text { vínculo não afetivo } 0 \text { (não descrito e profissional), } 1 \text { = vínculo afetivo } 1 \\
\text { (familiar ou amizade) }\end{array}$ \\
\hline \multicolumn{2}{|l|}{ Independentes } \\
\hline Gênero & $0=$ mulher, $1=$ homem \\
\hline Idade & Ano de 2019 menos data de nascimento \\
\hline Experiência & $0=$ sem experiência anterior ao crime, $1=$ com experiência anterior ao crime \\
\hline PreLíder & Presença de líder $=1$ de outra forma $=0$ \\
\hline PreGerente & Presença de posição gerencial $=1$ de outra forma $=0$ \\
\hline Aprop & Apropriação indébita $=1$ de outra forma $=0$ \\
\hline Corr & Corrupção $=1$ de outra forma $=0$ \\
\hline Eva & Evasão de divisas $=1$ de outra forma $=0$ \\
\hline Lava & Lavagem de dinheiro $=1$ de outra forma $=0$ \\
\hline Merc & Mercado negro de câmbio $=1$ de outra forma $=0$ \\
\hline Ocult & Ocultação de provas $=1$ de outra forma $=0$ \\
\hline Testa & Testa de ferro $=1$ de outra forma $=0$ \\
\hline Traf & Tráfico $=1$ de outra forma $=0$ \\
\hline
\end{tabular}

Fonte: Elaborada pelos autores.

Todos os modelos foram estimados pelo uso da regressão logística binária com base na estimação por máxima verossimilhança. Os modelos de regressão logística binária fazem parte do grupo de Modelos Lineares Generalizados, pois a variável dependente apresenta uma distribuição de Bernoulli (Fávero \& Belfiore, 2017).

\section{ANÁLISE DE DADOS}

\subsection{Análises de Correlação}

A Tabela 6 apresenta a matriz de correlação de Spearman para as variáveis do modelo que explicam as características dos líderes e dos gerentes. Na diagonal superior estão os coeficientes da variável Líder e na diagonal inferior os coeficientes da variável Gerencial. Segundo Gujarati e Porter (2011) quando os coeficientes apresentam correlações abaixo de 0,8 não há presença de multicolinearidade, assim, pode-se dizer que as variáveis não apresentem multicolinearidade.

Tabela 6. Correlações entre as variáveis dos modelos 1 e 2

\begin{tabular}{lcccr}
\hline & Gerencial & Gênero & \multicolumn{1}{c}{ Idade } & Experiência \\
\hline Líder & & -0.035 & 0.101 & $0.609 * * *$ \\
Gênero & 0.190 & & 0.184 & 0.130 \\
Idade & -0.049 & 0.184 & & $0.243^{*}$ \\
Experiência & $-0.334 * *$ & 0.130 & $0.243^{*}$ & \\
\hline Fonte: Elaborada pelos autores. & \\
Nota: *,**,** indica significância estatística a leveis de $0,10,0,05$ e 0,01, respectivamente.
\end{tabular}

Nota-se que Lider é positivamente correlacionado com Experiência. Ao contrário, Gerente é negativamente correlacionado com Experiência. Ainda, a variável Idade apresenta correlação positiva com Experiência. A Tabela 7 apresenta a matriz de correlação das variáveis dos modelos 3 e 4 . Na diagonal superior estão as correlações em relação à variável VíncSig e os coeficientes para a variável VíncAfe estão na parte inferior da diagonal. 
L. M. D. Maragno, P. de S. Knupp, J. A. Borba / Rev. Cont Org (2019) n. temático Fraudes e Corrupção: o que Contabilidade e Organizações têm a dizer? $12-18$

Tabela 7. Correlação entre as variáveis dos modelos 3 e 4

\begin{tabular}{|c|c|c|c|c|c|c|c|c|c|c|}
\hline & VincAfe & Aprop & Corr & Eva & Lava & Merc & Ocult & Testa & PreGerente & PreLíder \\
\hline VincSig & & 0.108 & $0.115^{*}$ & -0.109 & 0.059 & $-0.311 * * *$ & $0.298 * * *$ & $0.410 * * *$ & $-0.144 * *$ & 0.034 \\
\hline Aprop & 0.119 & & -0.050 & $-0.158 * *$ & 0.021 & $-0.162 * *$ & -0.050 & $-0.118^{*}$ & $0.226 * * *$ & 0.010 \\
\hline Corr & 0.034 & -0.050 & & -0.087 & -0.008 & -0.003 & 0.041 & -0.081 & $0.149 * *$ & 0.041 \\
\hline Eva & -0.077 & $-0.158 * *$ & -0.087 & & $-0.195 * * *$ & 0.080 & $-0.176^{* *}$ & -0.047 & -0.093 & $0.248 * * *$ \\
\hline Lava & 0.000 & 0.021 & -0.008 & $-0.195 * * *$ & & $-0.272 * * *$ & $-0.150 * *$ & 0.063 & $0.231 * * *$ & 0.105 \\
\hline Merc & $-0.183 * * *$ & $-0.162 * *$ & -0.003 & 0.080 & $-0.272 * * *$ & & $-0.180 * * *$ & $-0.219 * * *$ & -0.044 & 0.107 \\
\hline Ocult & $0.398 * * *$ & -0.050 & 0.041 & $-0.176 * *$ & $-0.150 * *$ & $-0.180 * * *$ & & -0.030 & $-0.149 * *$ & $-0.139 * * *$ \\
\hline Testa & $0.336 * * *$ & $-0.118^{*}$ & -0.081 & -0.047 & 0.063 & $-0.219 * * *$ & -0.030 & & $-0.121^{*}$ & 0.082 \\
\hline PreGerente & $-0.180 * * *$ & $0.226 * * *$ & $0.149 * *$ & -0.093 & $0.231 * * *$ & -0.044 & $-0.149 * *$ & $-0.121^{*}$ & & $-0.261 * * *$ \\
\hline PreLíder & 0.021 & 0.010 & 0.041 & $0.248 * * *$ & 0.105 & 0.107 & $-0.139 * *$ & 0.082 & $-0.261 * * *$ & \\
\hline
\end{tabular}

Fonte: Elaborada pelos autores.

Nota: $* * *, * * *$ indica significância estatística a leveis de $0,10,0,05$ e 0,01 , respectivamente 
Os coeficientes apresentam correlações abaixo de 0,8 , o que indica a não relação linear "perfeita" ou exata entre as variáveis independentes do modelo, ou seja, não há presença de multicolinearidade (Gujarati \& Porter, 2011). Observa-se que a variável VincSig é positivamente correlacionada a Corr, Ocult e Testa e negativamente correlacionada a Merc. Já a variável VíncAfe é positivamente correlacionado a Ocult e Testa e negativamente correlacionada a Merc e PreGerente.

\subsection{Análises dos modelos de regressão logística}

A Tabela 8 demonstra os resultados das estimativas de todos os modelos. O Painel A apresenta as estimativas dos modelos 1 e 2, em que as características dos líderes e dos gerentes são relacionadas para explicar a posição na organização criminosa.

Tabela 8. Modelos de regressão logística

\begin{tabular}{|c|c|c|c|c|c|c|}
\hline \multicolumn{7}{|c|}{ Painel A - Modelos 1 e 2} \\
\hline & \multicolumn{4}{|c|}{ Modelo 1 (Lider) } & \multicolumn{2}{|c|}{ Modelo 2 (Gerencial) } \\
\hline & Coef. & Erro padrão & $\mathrm{z}$ & Coef. & Erro padrão & $\mathrm{Z}$ \\
\hline Constante & -1.702 & 1.887 & -0.902 & -1.159 & 1.266 & -0.915 \\
\hline Gênero & -0.868 & 1.006 & -0.863 & 1.324 & 0.765 & $1.731^{*}$ \\
\hline Idade & -0.012 & 0.039 & -0.303 & -0.004 & 0.024 & -0.162 \\
\hline Experiência & 3.171 & 0.999 & $3.175 * * *$ & -3.097 & 1.534 & $-2.018 * *$ \\
\hline $\mathrm{N}$ & & & 51 & & & 51 \\
\hline $\mathrm{R}^{2}$ & & & 0.4065 & & & 0.245 \\
\hline $\mathrm{R}^{2}$ ajustado & & & 0.2324 & & & 0.1335 \\
\hline FIV médio & & & 1.16 & & & 1.00 \\
\hline AIC & & & 35.25 & & & 62.16 \\
\hline Chi2 & & & 45.86 & & & 41.24 \\
\hline Curva ROC & & & 0.829 & & & 0.728 \\
\hline \multicolumn{7}{|c|}{ Painel B - Modelos 3 e 4} \\
\hline & \multicolumn{4}{|c|}{ Modelo 3 (VincSig) } & \multicolumn{2}{|c|}{ Modelo 4 (VincAfe) } \\
\hline & Coef. & Erro padrão & $\mathrm{Z}$ & Coef. & Erro padrão & $\mathrm{Z}$ \\
\hline Constante & -1.455 & 0.461 & $-3.157 * * *$ & -2.761 & 0.547 & $-5.047 * * *$ \\
\hline Aprop & 2.696 & 0.868 & $3.107 * * *$ & 3.220 & 0.948 & $3.397 * * *$ \\
\hline Corr & 2.519 & 0.855 & $2.947 * * *$ & 1.406 & 0.917 & 1.533 \\
\hline Eva & 0.403 & 0.478 & 0.843 & 0.331 & 0.535 & 0.619 \\
\hline Lava & 0.739 & 0.459 & 1.611 & 0.574 & 0.507 & 1.132 \\
\hline Merc & -0.895 & 0.485 & $-1.843^{*}$ & 0.111 & 0.554 & 0.200 \\
\hline Ocult & 3.509 & 1.137 & $3.085 * * *$ & 4.797 & 1.164 & $4.121^{* * *}$ \\
\hline Testa & 2.290 & 0.438 & $5.225 * * *$ & 2.386 & 0.487 & $4.894 * * *$ \\
\hline PreGerente & -1.317 & 0.477 & $-2.759 * * *$ & -1.120 & 0.564 & $-1.987^{* *}$ \\
\hline PreLíder & -0.119 & 0.461 & -0.258 & 0.173 & 0.532 & 0.325 \\
\hline Observações & & & 193 & & & 193 \\
\hline $\mathrm{R}^{2}$ & & & 0.497 & & & 0.463 \\
\hline $\mathrm{R}^{2}$ ajustado & & & 0.439 & & & 0.3898 \\
\hline FIV médio & & & 1.30 & & & 1.37 \\
\hline AIC & & & 193.29 & & & 166 \\
\hline Chi2 & & & 193.09 & & & 164.49 \\
\hline Curva ROC & & & 0.878 & & & 0.861 \\
\hline
\end{tabular}

Fonte: Elaborada pelos autores.

Nota: *,***** indica significância estatística a leveis de $0,10,0,05$ e 0,01 , respectivamente. O Fator de Inflação da Variância (FIV) nos modelos 1 e 2 apresentaram valores entre 1.00 e 1.22. Nos modelos 3 e 4 apresentou valores entre 1.11 e 1.72 . O que indica fraca multicolinearidade. $\mathrm{O} \mathrm{R}^{2}$ não demonstra evidências de que a multicolinearidade pode estar influenciando as estimativas. Os testes do p-valor do chi2 para todos os modelos indicam que pode ser rejeitada a hipótese de que os modelos não são adequados. $\mathrm{R}^{2}$ e $\mathrm{R}^{2}$ ajustado de McFadden. 
O modelo 1 apresenta a variável dependente Líder positivamente relacionada a Experiência (coef. = $3.171, \mathrm{p}<0.01$ ). Isso significa que os líderes possuem experiência anterior ao crime, corroborando com o trabalho de Weerman (2003). O Modelo 2 apresenta a variável dependente Gerencial positivamente relacionada a Gênero (coef. $=1.324, \mathrm{p}<0.10)$ e negativamente relacionada a Experiência (coef. $=-3.097, \mathrm{p}<0,05$ ). Isso significa que os gerentes são em maioria homens, conforme mostrado no estudo de Dollar, Fisman e Gatti (2001). E não possuem experiência anterior no crime, o que corrobora com os achados de Van Mastrigt e Farrington (2011), os quais destacam que as habilidades e experiências do líder podem ser transmitidas para os cooptados.

Esses resultados, além de reforçarem a literatura de delinquência juvenil no sentido de que experiência anterior ao crime é um fator que define o líder da organização, contribuem demonstrando que a experiência é determinante em esquemas de corrupção e lavagem de dinheiro. Também, demonstram evidências de que homens são mais cooptados à função gerencial do que mulheres, contribuindo com os estudos de Bonny, Goode e Lacey (2015) e Dollar, Fisman e Gatti (2001). Ainda, os resultados evidenciam que os líderes e doleiros são os que possuem experiência anterior ao crime. Isso demonstra que, por terem experiência, não necessitam de pessoas com experiências anteriores ao crime em cargos gerenciais, visto que o modelo das características dos gerentes resultou em uma relação negativa para experiência.

Os modelos 3 e 4 presentes no Painel B apresentam as estimativas dos tipos de crimes e a presença de líderes e gerentes (verificada nos modelos 1 e 2) em relação à natureza do vínculo entre fraudadores, proposto por Free e Murphy (2015). No Modelo 3, a variável dependente é VíncSig demonstrou-se positivamente relacionada a Apro (coef. $=2.696, \mathrm{p}<0.01)$, Corr $($ coef. $=2.519, \mathrm{p}<0.01)$, Ocult $($ coef. $=3.509, \mathrm{p}<0.01)$ e Testa $($ coef. $=$ $2.290, \mathrm{p}<0.01)$. Ainda, negativamente relacionada a Merc (coef. $=-0.895, \mathrm{p}<0.10)$ e PreGerente $($ coef. $=-1.317$, $\mathrm{p}<0.01)$.

Isso significa que para os crimes de apropriação, corrupção, ocultação e testa de ferro, é necessário algum tipo de vínculo significativo entre fraudadores, como profissional, familiar ou de amizade. Ainda, esses resultados demonstraram que quando pelo menos uma das partes está no nível gerencial da organização criminosa o vínculo pessoal entre os indivíduos tende a ser não afetivo, ou seja, nesse nível hierárquico as pessoas se relacionam mais com indivíduos com os quais não possuem laços familiares ou de amizade. Sugerindo que as relações são construídas após a cooptação do ato e assim, desenvolvidas ao longo do tempo de forma restrita ao vínculo profissional.

Os resultados para presença de Líder - aquele que comanda a organização criminosa -, apresentaram relação negativa para vínculos significativos. Esses resultados sugerem que o esquema pode ter se tornado muito grande e são necessárias relações do líder com diversas pessoas na organização, além de seus familiares, amigos e profissionais. Também, como explicação alternativa, pode ser que os líderes possam preferir preservar a família e, consequentemente, evitar conflitos afetivos (Free \& Murphy, 2015).

No Modelo 4, a variável dependente VíncAfe, demonstrou-se positivamente relacionada a Aprop (coef. $=3.220, \mathrm{p}<0.01)$, Ocult (coef. $=4.797, \mathrm{p}<0.01)$, Testa (coef. $=2.386, \mathrm{p}<0.01)$. Isso significa que além do vínculo ser significativo, o mesmo é afetivo (isto é, família ou amigos). Ainda, a variável dependente apresentase negativamente relacionada a PreGerente (coef. $=-1.120 \mathrm{p}<0.05$ ). Ou seja, os vínculos afetivos (familiar ou amizade) se relacionam positivamente para Apropriação Indébita, Ocultação de provas e Testa de Ferro.

Tanto no Modelo 3 quanto no Modelo 4 a PreGerente apresentou relação negativa e estatisticamente significativa. Isso implica que as pessoas em cargos gerenciais não são aquelas que apresentam relações com vínculos funcionais ou afetivos (família ou amizade).

Fee e Murphy (2015) destacaram a natureza dos vínculos entre membros de um grupo fraudulento e, nossos resultados fornecem evidências empíricas para os vínculos funcionais que servem à organização e para os vínculos afetivos.

Em relação à Apropriação Indébita foi observado que um político corrupto se associava a um doleiro para, por meio de uma empresa de fachada, movimentar e lavar o dinheiro adquirido da corrupção. O crime de ocultação de provas se refere à cooptação de familiares, para ocultar provas. Sobre Testa de Ferro, os resultados demonstraram relações positivas entre vínculos afetivos e Testa de Ferro. Esse resultado sugere que a confiança em membros da família ou amigos é necessária para que o fraudador empreste o nome (isto é, CPF) para que empresas de fachada sejam criadas e, ainda, realizem transações financeiras. 


\section{CONCLUSÃO}

A compreensão do fenômeno do conluio, especialmente em esquemas de corrupção e lavagem de dinheiro é limitada e, ainda, Dimant e Tosato (2018) alegam um esforço recente para necessidade de compreender a natureza multifacetada da corrupção e suas interações no nível micro, meso e macro. Portanto, esse artigo exploratório buscou expandir os achados, apresentando evidências a partir do caso relacionado à Operação "Lava Jato". A base de dados construída permitiu expor evidências sobre com quem os líderes das organizações criminosas decidiram cofraudar e quais suas características. $\mathrm{O}$ artigo apresenta evidências sobre as características dos cofraudadores em posições gerencias na hierarquia da organização criminosa. Por fim, o trabalho buscou explicar a natureza dos vínculos entre fraudadores e cofraudadores e suas relações aos tipos de crimes.

Os resultados empíricos demonstraram que a corrupção foi realizada não somente por pessoas que possuíam relações de afetividade/amizade, mas também relações de trabalho que potencializam e podem dificultar a identificação de ligações suspeitas entre criminosos pelos órgãos de controle (PF e MPF). Neste sentido, o aperfeiçoamento dos órgãos de inteligência é sintomático, visto que a corrupção gera demandas diversas e pode estar correlacionada com outros crimes financeiros, seja para lavar dinheiro, operação ilegal de câmbio ou evasão de divisas, dentre outras.

Uma das formas predominantes identificadas na Operação "Lava Jato" para a prática de crimes foi a utilização de "testas de ferro" que colaboram na transformação de recursos ilícitos em "aparentemente" lícitos. A tônica tem sido a aquisição de bens, a realização de transações financeiras fictícias e o gerenciamento de empresas de fachada com objetivo de lavar o dinheiro obtido. Diferentemente dos "laranjas", que eventualmente são pessoas usadas sem sua ciência, para simular atos criminosos, os "testas de ferro" são elementos preponderantes nas atividades criminosas de lavagem de dinheiro. Dessa forma, o Código Penal poderia tipificar tais condutas, que invariavelmente representam falsamente a propriedade de empresas, cedem a titularidade de contas bancárias e/ou colocam veículos e outros bens em seus nomes.

As limitações do trabalho proporcionam oportunidades de trabalhos futuros. A primeira limitação se refere à coleta de dados, somente foram lidos os processos referentes à primeira fase da Operação "Lava Jato", que tem seu foco nos líderes dos esquemas. Trabalhos futuros podem expandir para as demais fases e/ou outras investigações. Ainda, neste trabalho não foram identificados quando os cofraudadores foram cooptados, ou seja, quando se deu o início a relação. Outra limitação foi assumir que quando o vínculo não era descrito ele não era significativo, assim, entrevistas com os acusados podem suprir essa limitação.

Trabalhos futuros podem explorar, ainda, cada crime individualmente, principalmente os mais frequentes no caso "Lava Jato" (lavagem de dinheiro, evasão de divisas e mercado negro de dinheiro), a exemplo de Gonçalves \& Andrade (2019) que abordaram a corrupção em específico. Por fim, os vínculos funcionais que servem ao indivíduo proposto por Free e Murphy (2015), ou seja, os motivos individuais de cada acusado não foram coletados devido a leitura dos processos não permitir identificação de motivos pessoais aos quais os indivíduos decidiram cofraudar.

\section{REFERÊNCIAS}

ACFE (2018). Report to the nations. Disponível em: $<$ https://www.acfe.com/report-to-the-nations/2018/> Acesso em: 15 maio 2019.

Alvarez, J. P. (2015). Low-Level Corruption Tolerance: An “Action-Based” Approach for Peru and Latin America. Journal of Politics in Latin America, 7(2), 99-129. DOI: https://doi.org/10.1177/1866802X1500700204.

Ashforth, B. E., \& Anand, V. (2003). The normalization of corruption in organizations. Research in organizational behavior, 25, 1-52. DOI: https://doi.org/10.1016/S0191-3085(03)25001-2.

Bishop, C. C., Hermanson, D. R., \& Riley Jr, R. A. (2017). Collusive fraud: Leader, incident, and organizational characteristics. Journal of Forensic Accounting Research, 2(1), 49-70. DOI: https://doi.org/10.2308/jfar51826.

Bonny, P., Goode, S., \& Lacey, D. (2015). Revisiting employee fraud: gender, investigation outcomes and offender motivation. Journal of Financial Crime, 22(4), 447-467. DOI: https://doi.org/10.1108/JFC-04-2014-0018.

Button, M., Shepherd, D., \& Blackbourn, D. (2018). Co-offending and bribery: the recruitment of participants to corrupt schemes and the implications for prevention. Security Journal, 31, 882-900. 
Chaikin, D. (2008). Commercial corruption and money laundering: a preliminary analysis. Journal of Financial Crime, 15(3), 269-281. DOI: https://doi.org/10.1108/13590790810882865.

Cox, D. (2014). Handbook of anti-money laundering. John Wiley \& Sons.

Dimant, E., \& Tosato, G. (2018). Causes and effects of corruption: what has past decade's empirical research taught us? A survey. Journal of Economic Surveys, 32(2), 335-356. DOI: https://doi.org/10.1111/joes.12198.

Dollar, D., Fisman, R., \& Gatti, R. (2001). Are women really the "fairer" sex? Corruption and women in government. Journal of Economic Behavior \& Organization, 46(4), 423-429. DOI: https://doi.org/10.1016/ S0167-2681(01)00169-X.

Fávero, L.P., \& Belfiore, P. (2017). Manual de Análise de Dados: Estatística e Modelagem Multivariada com excel, SPSS e Stata. Elsevier, Rio de Janeiro.

Free, C. (2015). Looking through the fraud triangle: A review and call for new directions. Meditari Accountancy Research, 23(2), 175-196. DOI: https://doi.org/10.1108/MEDAR-02-2015-0009.

Free, C., \& Murphy, P. R. (2015). The ties that bind: The decision to co-offend in fraud. Contemporary Accounting Research, 32(1), 18-54. DOI: https://doi.org/10.1111/1911-3846.12063.

Gonçalves, V. B., \& Andrade, D. M. (2019). A corrupção na perspectiva durkheimiana: um estudo de caso da Operação Lava Jato. Revista de Administração Pública. (53)2, 271-290. DOI: http://dx.doi.org/10.1590/0034761220180192.

Jancsics, D. (2019). Corruption as resource transfer: An interdisciplinary synthesis. Public Administration Review. (79)4, 523-537. DOI: https://doi.org/10.1111/puar.13024.

Kleemans, E. R., \& De Poot, C. J. (2008). Criminal careers in organized crime and social opportunity structure. European Journal of Criminology, 5(1), 69-98.

Kominis, G., \& Dudau, A. (2018). Collective corruption-How to live with it: Towards a projection theory of post-crisis corruption perpetuation. European Management Journal, 36(2), 235-242. DOI: https://doi. org/10.1016/j.emj.2017.12.001.

Lantz, B., \& Hutchison, R. (2015). Co-offender ties and the criminal career: The relationship between co-offender group structure and the individual offender. Journal of Research in Crime and Delinquency, 52(5), 658-690. DOI: https://doi.org/10.1177/0022427815576754.

Levi, M., \& Reuter, P. (2006). Money laundering. Crime and Justice, 34(1), 289-375. DOI: https://doi. org/10.1086/501508.

Maragno L. M. D., \& Borba, J. A. (2019). Unearthing money laundering at Brazilian Oil Giant Petrobras. Journal of Money Laundering Control. 22(2). DOI: https://doi.org/10.1108/JMLC-03-2018-0027.

Maragno, L. M. D., \& Borba, J. A. (2017). Mapa conceitual da fraude: configuração teórica e empírica dos estudos internacionais e oportunidades de pesquisas futuras. Revista de Educação e Pesquisa em Contabilidade, 11, 41-68. DOI: https://doi.org/10.17524/repec.v11i0.1665.

Mclannahan, B. (2017). Fiscalização em bancos globais afeta emergentes. Valor Econômico. São Paulo. Disponível em: $\quad<$ https://www.valor.com.br/financas/5113464/fiscalizacao-em-bancos-globais-afeta-emergentes>. Acesso em: 15 maio 2019.

Mcgloin, J. M., \& Nguyen, H. (2012). It was my idea: Considering the instigation of co-offending. Criminology, 50(2), 463-494. DOI: https://doi.org/10.1111/j.1745-9125.2011.00266.x.

Milne, R. (2019). Prosecutors charge ex-Danske Bank chief in money-laundering probe: Thomas Borgen is first senior manager to be indicted in €200bn scandal. Financial Times. Londres, 07 maio 2019. Disponível em: $<$ https://www.ft.com/content/a78b04ba-70d5-11e9-bf5c-6eeb837566c5>. Acesso em: 13 maio 2019.

OCDE. (2018). Money laundering. Disponível em: < https://www.oecd.org/cleangovbiz/toolkit/moneylaundering. $\mathrm{htm}>$ Acesso em: 13 maio 2019.

Pinto, J., Leana, C. R., \& Pil, F. K. (2008). Corrupt organizations or organizations of corrupt individuals? Two types of organization-level corruption. Academy of Management Review, 33(3), 685-709. DOI: https://doi. org/10.5465/amr.2008.32465726. 
Reuters (2016). IMF: Global corruption costs trillions in bribes, lost growth. Disponível em: <https:// www.reuters.com/article/us-imf-corruption/imf-global-corruption-costs-trillions-in-bribes-lost-growthidUSKCN0Y22B7>. Acesso em: Acesso em: 15 maio 2019.

Schwartzkopff, F., \& Magnusson, N. (2019). Swedbank Hunts for CEO as Dirty Money Investigations Continue. Bloomberg. Nova York. Disponível em: < https://www.bloomberg.com/news/articles/2019-05-13/swedbankhunts-for-ceo-as-dirty-money-investigations-continue>. Acesso em: 15 maio 2019.

Tillyer, M. S., \& Tillyer, R. (2015). Maybe I should do this alone: A comparison of solo and co-offending robbery outcomes. Justice Quarterly, 32(6), 1064-1088. DOI: https://doi.org/10.1080/07418825.2014.883422.

Trompeter, G. M., Carpenter, T. D., Jones, K. L., \& Riley Jr, R. A. (2014). Insights for research and practice: What we learn about fraud from other disciplines. Accounting Horizons, 28(4), 769-804. DOI: https://doi. org/10.2308/acch-50816.

UNODC. (2019). Money-Laundering and Globalization. Disponível em: <https://www.unodc.org/unodc/en/ money-laundering/globalization.html>. Acesso em: 10 abril 2019

Van Mastrigt, S. B., \& Farrington, D. P. (2011). Prevalence and characteristics of co-offending recruiters. Justice Quarterly, 28(2), 325-359. DOI: https://doi.org/10.1080/07418825.2010.482535.

Weerman, F. M. (2003). Co-offending as a social exchange: Explaining characteristics of co-offending. British Journal of Criminology, 43, 398-416. DOI: https://doi.org/10.1093/bjc/43.2.398. 
Apêndice A

Exemplo do Vínculo Afetivo no Processo Penal: 5025676-71.2014.404.7000 entre o fraudador (Paulo Roberto Costa) e co-fraudadora (Shanni Azevedo Costa Bachmann)

Shanni Azevedo Costa Bachmann, nascida em 13/08/1981, filha de Paulo Roberto Costa e Marici da Silva Azevedo Costa - CPF: 091.878.667-30, Endereço: Rua dos Jacarandás, 1000, Bloco 03, Apto 501 - Barra da Tijuca - Rio de Janeiro/RJ - CEP 22.776-050

Exemplo do papel no crime do Processo Penal: 5025676-71.2014.404.7000 da co-fraudadora (Shanni Azevedo Costa Bachmann)

Já o casal HUMBERTO e SHANNI chegou no escritório às 9 horas e 20 minutos. HUMBERTO permaneceu no local somente até as 9 horas e 24 minutos, quando deixou o prédio e passou a esperar do lado de fora. Durante todo o tempo, o denunciado demonstrou ter consciência dos fatos e prestou auxílio material aos demais denunciados.

A denunciada SHANNI, por sua vez, permaneceu até 9 horas e 53 minutos no escritório prestando auxílio na remoção de documentos para o denunciado MARCIO. Durante este período subiu e desceu os elevadores por duas vezes. A primeira vez com uma bolsa de ginástica e uma bolsa preta pessoal. A segunda, apenas com a bolsa preta pessoal.

Assim, coube aos denunciados ARIANA E MARCIO a tarefa de remover materialmente as provas dos crimes praticados pela organização criminosa. Já os denunciados HUMBERTO e SHANNI prestaram auxílio material sem o qual a empreitada criminosa não seria consumada. Enquanto SHANNI auxiliava MARCIO na identificação e remoção dos documentos de dentro do escritório, HUMBERTO, prestou auxílio aguardando no carro do lado de fora.

Todos atenderam a ordem do denunciado PAULO ROBERTO COSTA, que era o único interessado na destruição das provas.

Exemplo papel no crime no Processo Penal: 5049898-06.2014.404.7000 do co-fraudador (Carlos Alberto Pereira da Costa)

No esquema, MURILO TENA BARRIOS, junto com Marcio Bonilho, era diretor das empresas Sanko Sider e Sanko Serviços, envolvidas no esquema. CARLOS ALBERTO PEREIRA DA COSTA atuava como "testa de ferro", mas também auxiliava no planejamento jurídico das atividades e na lavagem de valores, sobretudo administrando as empresas de YOUSSEF.

Exemplo de experiência no Processo Penal: 5026212-82.2014.404.7000 do fraudador (Alberto Youssef)

A atuação de YOUSSEF data de longa data no sistema financeiro paralelo. Trabalha, no mínimo, há vinte anos no mercado de câmbio paralelo, como doleiro, e já foi considerado um dos maiores doleiros do Brasil. Conforme é sabido, foi condenado pela Justiça pela evasão de divisas. Interessante anotar diálogo em que YOUSSEF conversa com ANDRÉ LUIS e afirma que já chegou a ter 150 milhões de dólares na conta, antes de "quebrar" - ou seja, de ser preso no passado - e mesmo assim pagou todo mundo.

Exemplo de experiência no Processo Penal: 5026663-10.2014.404.7000 do fraudador (Carlos Habib Chater). HABIB já foi condenado pela Justiça Federal do Distrito Federal, em duas ações penais pela prática de crimes contra o sistema financeiro e pelo comércio ilegal de moeda estrangeira, bem como constituição de sociedades em nome de laranjas, condutas que estão agora novamente sendo-lhe imputada, o que denota que faz dessas práticas o seu meio de vida. 